\title{
1 Topographically organized representation of space and context in the medial
}

\section{2 prefrontal cortex}

3 Jonas-Frederic Sauer*, Marlene Bartos*

4 University of Freiburg, Medical Faculty, Institute of Physiology I, Hermann-Herder-Strasse 7, D-

579104 Freiburg, Germany.

6 *Correspondence: marlene.bartos@physiologie.uni-freiburg.de, jonas.sauer@physiologie.uni-

7 freiburg.de 


\section{Abstract}

9 Spatial tuning of pyramidal cells has been observed in diverse neocortical regions, but a systematic

10 characterization of the properties of spatially tuned neurons across cortical layers and regions is

11 lacking. Using mice navigating through virtual environments, we find topographical organizational

12 principles for the representation of spatial features in the medial prefrontal cortex. We show that

13 spatial tuning emerges across layers with a dorso-ventral gradient in the depth of spatial tuning,

14 which resides in superficial layers. Moreover, the prefrontal cortex shows hemispheric lateralization

15 of spatial tuning such that neurons located in the left hemisphere display more pronounced spatial

16 tuning. During exploration of a novel compared to a familiar context, a different picture emerges.

17 Context discrimination and familiarity detection is higher in superficial compared to deep layers.

18 However, neurons of the right medial prefrontal cortex discriminate more efficiently between

19 contexts than cells in the left hemisphere. Jointly, these results reveal a complex topographic

20 organization of spatial representation and suggest a division of labor among prefrontal layers and

21 subregions in the encoding of spatial position in the current environment and context

22 discrimination.

\section{Significance}

25 The neocortex is composed of areas with specialized function (e.g. sensory vs. associational).

26 Despite this functional diversity, emerging evidences suggest that the encoding of space might be a

27 universal feature of cortical circuits. We identified distinct gradients of spatial tuning depth along

28 the dorso-ventral and radial intrahemispheric and left-right interhemispheric axes. Moreover,

29 neurons located in superficial layers in the right hemisphere show more pronounced context

30 differentiation between a familiar and a novel environment. A complex topography of spatial tuning

31 properties and context differentiation might thus support division of labor among medial prefrontal 
32 cortical subnetworks to support local network computation relevant for the execution of context-

33 dependent behavioral outcomes.

\section{Introduction}

36 Encoding of spatial information is classically assigned to the hippocampal-entorhinal system (1) (2)

37 (3). However, with recent reports of spatially tuned neurons in the posterior parietal (4), 38 retrosplenial (5), visual (6), and somatosensory cortex (7), as well as over large areas of the dorsal

39 cortex including sensory and higher-order areas (8), it is becoming evident that spatially modulated

40 activity is a more widespread phenomenon than initially assumed and might be a universal feature seen across the neocortex.

The medial prefrontal cortex (mPFC) is critical for cognitive functions such as the planning and execution of complex behaviors (9). Many cognitive tasks involving the mPFC require the

44 simultaneous awareness of spatial variables including one's own current position in an environment,

45 the recognition of novelty/familiarity of the surrounding features, and context discrimination (10)

46 (11). In line with this, prefrontal neurons of mice display spatially tuned activities during cognitive 47 tasks (12) (13) (14) (15) (16).

However, compared to the extensively studied properties of spatial coding in the

49 hippocampal-entorhinal system it remains largely unclear whether mPFC neurons display spatially

50 tuned activities across layers and subfields (17) as recently demonstrated for the retrosplenial cortex

51 (5). Indeed, in the retrosplenial cortex spatial tuning is more pronounced in superficial than deep

52 layers (5), whereas in the somatosensory cortex, spatially tuned neurons are prominently observed 53 in deep layers (7), although systematic comparisons among layers are lacking. Distinct neocortical 54 subfields furthermore receive different afferent inputs, including those from the hippocampal 55 formation (18) (19), thus varying degrees of spatial tuning across subareas and layers might be 56 expected. Furthermore, while the hippocampal place code rapidly emerges during exploration of 
57 environments (20) (21), it is not clear how fast the neocortical spatial map is established once the

58 animal is introduced to an arena.

$59 \quad$ Here, we addressed these questions by assessing spatial tuning in the mPFC across layers

60 and subfields along the dorso-ventral axis while mice were navigating through virtual

61 environments. We find that spatial tuning emerges rapidly upon context exposure, even in a novel

62 arena. Moreover, we find pronounced topographical gradients of spatial modulation depth along the

63 dorso-ventral and left right axes and a differential contribution of prefrontal layers to context

64 differentiation. These data thus reveal a complex organization of neocortical spatial tuning

65 properties along multiple prefrontal axes.

\section{Results}

68 Prefrontal neurons represent space during spontaneous unrewarded navigation

69 We trained head-fixed mice to run consecutive laps on a virtual circular track (1.5 or 2 m long; Fig.

70 1). The track was composed of linear segments with distinctive wall patterns and additional cues

71 located outside the track (Fig. 1A). We chose this experimental setting to constrain the assessment

72 of spatial tuning in one dimension (i.e. 1-D position on the track) and to avoid complex behaviours

73 such as grooming or rearing, which are observed in freely moving mice and modulate the activity of

74 prefrontal neurons (22). The animals moved along the track in a self-induced and self-paced manner

75 without rewards. After familiarization to the track for several days, we recorded single units with

76 linear silicon probes acutely lowered into the mPFC (Fig. 1B). To assess spatial representation, we

77 quantified two parameters, sparsity and spatial information, from spatially binned firing rates of

78 each neuron (8) (12). Sparsity quantifies the proportion of bins with average firing of at least 25\%

79 of the peak rate of each unit. Low values of this metric thus indicate that spiking activity is spatially

80 confined to few bins on the track (Fig. 1D). 
In total, we recorded from 1800 prefrontal neurons ( $N=17$ mice). Based on spike waveform

82 kinetics, we grouped the cells in putative pyramidal $(N=1543)$ and GABAergic interneurons

83 ( $N=257$, SI Appendix, Fig. S1). Analysis of sparsity and spatial information during movement

84 revealed a broad distribution for the pyramidal cell population, with many units displaying spatially confined activity (Fig. 1C,D). Both parameters were significantly different from a surrogate dataset created by shuffling the spike trains of each unit, indicating above-chance spatial representation by the population of prefrontal pyramidal neurons during spontaneous locomotion (Sparsity: $P=10^{-55}$; Spatial information: $P=1.5^{*} 10^{-21}$; Fig. 1D). Consistent with previous reports in spatial memory tasks (12), spatial tuning of prefrontal pyramidal units was lower than in the hippocampus under our experimental conditions ( $P=0.002$; Fig. 1E). The firing of putative interneurons contained significant spatial information but spatial tuning was lower than in pyramidal cells (SI Appendix, Fig. S1). Further analyses showed significant stability of the spatial map within the recording session (SI Appendix, Fig. S2). In addition, spatial tuning was evident in population data extracted with principal component analysis (SI Appendix, Fig. S3).

To investigate the time course with which the spatial map emerged, we correlated for each pyramidal cell the average spatial tuning function with the spatially binned activity during individual runs (Fig. 1F). This analysis indicated a rapid onset of spatial tuning upon exposure to the virtual environment from the second run onwards. The activity of prefrontal neurons, particularly of pyramidal neurons, thus contains information about the position of the animal during spontaneous self-paced motion.

\section{Spatial tuning emerges across prefrontal layers}

103 To test whether spatial tuning properties differ among cortical layers, we quantified the laminar 104 position of the silicon probes in the mPFC (Fig. 2A). During 34 recording sessions from 17 mice, 
session did not depend on laminar depth (Fig. 2B, sparsity: $P=0.621$, spatial information: $P=0.257$, recording depth ranged from 199 to $714 \mu \mathrm{m}$ ). Both layer 2/3 and layer 5/6 pyramidal cells were spatially tuned as they displayed significant sparsity (layer 2/3: $P=1.6^{*} 10^{-11}, N=233$ cells, 4 mice; layer 5/6: $P=1.3^{*} 10^{-8}, N=311$ cells, 7 mice, recordings from the left hemisphere) and spatial information content (layer 2/3: $P=0.0004$, layer 5/6: $P=0.002$, Fig. 2C) compared to shuffled controls. Comparisons of sparsity of cells recorded in layer 2/3 with those recorded in layer 5/6 revealed no significant difference ( $P=0.112$, Fig. 2C). However, we found higher average spatial information in layer $2 / 3$ neurons $(P=0.03$, Fig. 2C). Since sparsity and spatial information both

114 showed an inverse correlation with firing rate, unequal firing rates might confound the comparison of spatial tuning properties among cortical layers (SI Appendix, Fig. S4). Indeed, layer 2/3 neurons discharged at lower average rates during movement on the track than layer 5/6 cells (layer 2/3: 2.4 \pm $0.2 \mathrm{~Hz}$, layer 5/6: $3.3 \pm 0.3 \mathrm{~Hz}, P=0.007$, Fig. 2D). We therefore examined whether distinct spatial tuning properties persist when we consider a population of neurons with equal firing rate. To address this question, we performed an iterative procedure in which a random subset of layer 5/6 neurons was drawn from the total population of cells, and spatial parameters were compared to the

121 layer 2/3 population only if the two groups showed similar mean firing rates during running on the 122 virtual maze ( $P>0.05$ for the comparison of rates; Methods). In 1000 rate-matched iterations, no 123 cases of significantly different sparsity were observed, and spatial information showed a significant 124 difference in only 35.1\% of iterations (Fig. 2E). Thus, our analysis indicates that comparisons of superficial and deep layer neuronal populations with matching firing rate show similar spatial 126 information contents.

To further rule out layer-specific spatial modulation, we performed simultaneous recordings 128 at two cortical depths using multi-shank probes (shank spacing $250 \mu \mathrm{m}$, Fig. 2F,G). Sparsity, spatial 129 information and firing rate did not depend on cortical depth in these recordings (sparsity: $P=0.629$, 130 spatial information: $P=0.204$, rate: $P=0.563$, pairwise comparison between simultaneously recorded 
131 superficial and deep shanks, $N=6$ sessions from 3 mice, Fig. 2G). These data jointly show similar

132 spatial tuning across cortical layers of the mPFC.

134 A dorso-ventral gradient of spatial representation

135 Hippocampal inputs transfer spatial information to the mPFC network (10). Tracing studies

136 indicated that hippocampal projections to the mPFC show the highest density in ventral medial 137 prefrontal areas (23) (24) (25) (26). We therefore hypothesized that spatial tuning of mPFC neurons 138 might depend on their position along the dorso-ventral axis of the mPFC (Fig. 3). Linear silicon 139 probes allow pinpointing the location of the recorded units based on the amplitude of the 140 extracellular waveform on neighboring electrodes, enabling us to determine the position of each 141 recorded pyramidal neuron along the dorso-ventral axis (ranging from accessory motor 142 cortex/cingulate cortex to prelimbic/medial orbital cortex). Consistent with hippocampal 143 projections, we found a higher power of theta $(6-12 \mathrm{~Hz})$ oscillations in ventral mPFC areas 144 (Spearman's $r=-0.995, P=10^{-63}$, Fig. 3B,C). Unexpectedly, when we binned the neurons in those 145 located on the top or bottom $300 \mu \mathrm{m}$ of the shank (corresponding to accessory motor/cingulate cortex and prelimbic/medial orbital cortex, respectively, Fig. 3D), we found lower sparsity of units

147 belonging to the dorsal group ( $P=0.0002, N=337$ dorsal and 265 ventral neurons; Fig. 3E). There 148 was furthermore a trend towards higher spatial information content of dorsal neurons, which did not 149 reach significance $(P=0.094$; Fig. $3 E)$. Firing rate $(P=0.326)$ and strength of coupling to theta 150 oscillations $(P=0.7)$ did not differ between dorsal and ventral neurons. Thus, our data suggest 151 stronger spatial tuning of neurons in the dorsal mPFC based on their sparsity. speculated that layer-dependent differences might attenuate potential dorso-ventral gradients in 154 spatial tuning. We therefore separately analyzed layer 2/3 ( $N=125$ dorsal and 104 ventral neurons) 155 and layer 5/6 cells ( $N=212$ dorsal and 161 ventral neurons, Fig. 3F-K). In superficial layers, we 
156 detected a prominent dorso-ventral gradient in sparsity and spatial information, with stronger spatial

157 tuning in the dorsal mPFC (Sparsity: $P=6.4 * 10^{-6}$, Spatial information: $P=0.007$, Fig. 3F). Since

158 layer 2/3 cells in the dorsal mPFC showed higher firing rates than the ones in the ventral group

159 ( $P=0.011$ ), we applied the rate-matching iteration procedure. This analysis revealed a significant

160 difference between dorsal and ventral neurons in sparsity in $100 \%$ and in spatial information in $78 \%$

161 of rate-matched comparisons (1000 iterations, Fig. 3G), further confirming higher spatial tuning of

162 dorsal mPFC cells. In agreement with the observed dorso-ventral gradient in spatial tuning depth,

163 dorsally located neurons in superficial layers showed significantly stronger theta-coupling

164 compared to ventrally located cells ( $\mathrm{P}=0.035$, Fig. $3 \mathbf{H})$. In contrast, recordings from deep layers

165 showed no difference in sparsity $(P=0.176)$ or spatial information $(P=0.972)$ along the dorso-ventral

166 axis (Fig. 3I,J). In contrast to layer 2/3 neurons, ventrally located cells in deep layers were more

167 strongly entrained by theta activity patterns than superficial neurons ( $P=0.003$, Fig. 3K). Jointly,

168 these data point to a dorso-ventral gradient in spatial tuning depth of cells in superficial layers of the 169 mPFC.

\section{Hemispheric asymmetry of spatial representation}

172 The observed dorso-ventral gradient in spatial modulation depth motivated us to search for

173 additional topographical rules of spatial representation. Prefrontal cognitive functions in humans are

174 highly lateralized (27) (28) (29). Therefore, we examined whether the properties of spatial

175 representation might differ between the left and right mPFC (Fig. 4). We observed significantly

176 lower sparsity $\left(P=8.9 * 10^{-22}\right)$ and higher spatial information in neurons recorded in the left compared

177 to the right mPFC $\left(P=4.4^{*} 10^{-13}, N=544\right.$ and 639 neurons from 11 and 8 mice in left and right

178 mPFC, respectively; data were pooled across layers; Fig. 4A). There was furthermore a lower

179 average firing rate in left prefrontal neurons ( $P=0.008$, Fig. 4A). Rate-matching analysis revealed

180 that the effects of lower sparsity and higher spatial information were also evident when accounting 
181 for rate differences in 100\% of iterations (Fig. 4B). Furthermore, theta-coupling depth was higher in

182 the left versus the right mPFC ( $P=0.004$; Fig. 4C). Thus, our data indicate stronger spatial tuning in 183 the left hemisphere.

Since we identified dorso-ventral differences in spatial tuning in superficial but not deep

layers (Fig. 3F), we restricted our further analysis to recordings from layers 5/6 ( $N=311$ and 639

neurons from 7 and 8 mice for left and right mPFC, respectively). Similar to the full dataset, this analysis showed lower sparsity $\left(P=1.8^{*} 10^{-12}\right)$, higher spatial information $\left(P=4.5^{*} 10^{-7}\right)$ and stronger theta entrainment in the left mPFC ( $P=0.046)$, while firing rates were similar $(P=0.407$; Fig. 4D,E).

To rule out the potential influence of inter-individual differences, we further restricted our analysis

subsequent days ( $N=142$ and 213 neurons from 3 mice). Again, sparsity was lower $\left(P=1.2^{*} 10^{-14}\right)$, while spatial information $\left(P=5.3^{*} 10^{-6}\right)$ and theta coupling depth were higher in the left compared to the right $\mathrm{mPFC}\left(P=2.7^{*} 10^{-5}\right.$; Fig. $\left.\mathbf{4 F}, \mathbf{G}\right)$. Thus, these data indicate interhemispheric asymmetries with pyramidal neurons in the left mPFC displaying more prominent spatial tuning properties than the ones in the right mPFC.

\section{Topographical gradients in representational stability}

Finally, we assessed context differentiation and stability of spatial tuning by sequentially exposing mice to a familiar (fam) and an additional novel unfamiliar circular track (nov), and compared the data with a re-exposure to the same familiar (fam’) track within one recording session (Fig. 5). The novel track differed from the familiar environment in size (track length $3 \mathrm{~m}$ ) and visual features (colors, wall patterns, external cues, Fig. 5A). We previously demonstrated that mice are capable to recognize such novel virtual tracks (30). Analysis of waveform parameters indicated stable

204 recording conditions throughout the exposure to the three environments (SI Appendix, Fig. S5).

205 Since spatial tuning of prefrontal neurons during exposure to a novel track has not been 
characterized, we first quantified the spatial tuning properties of all pyramidal neurons irrespective of their anatomical position. Pyramidal neurons showed significant sparsity and spatial information compared to shuffled datasets in all three conditions (Fig. 5C), indicating spatial tuning on the novel track. The neurons displayed comparable average firing rates during running in all three experimental conditions (SI Appendix, Fig. S5). To assess remapping of pyramidal neurons during exposure to novelty, we quantified the correlation coefficient of the mean occupation-normalized spatially binned firing rates for each neuron (spatial stability). Spatial stability between fam and nov was low (mean correlation $0.048 \pm 0.008, P<0.05$; Fig. 5D). Given that spatial tuning is observed in

214 both fam and nov conditions (Fig. 5C), these data suggest that exposure to a novel environment leads to a rearrangement of spatial representation in the mPFC. During exploration of nov, correlation of the spatial tuning function during individual runs to the average map of each neuron revealed that spatial tuning was significantly different from shuffled data from the second lap onwards (Fig. 5E). Spatial representation thus rapidly remaps during exploration of the novel track. During re-exposure to fam', we found a much higher correlation to the original spatial pattern observed in fam (mean correlation: $0.210 \pm 0.01, P<0.05$; Fig. 5D), indicating that the spatial representation of the original environment is partially reinstated upon re-exposure to the same arena. Consistent with this observation, analysis of spatially binned population dynamics revealed a higher spatial correlation between fam and fam’ compared to fam and nov (SI Appendix, Fig. S5). (i.e. a lower correlation of the spatial tuning function) between fam and nov in superficial compared to deep layer neurons (Fig. 5F, $P=0.047, N=162$ layer 2/3 and 142 layer 5 pyramidal neurons; we restricted this analysis to recordings from the left hemisphere to exclude inter-hemispheric 
231 higher in layer 2/3 $(P=0.0003$, Fig. 5F). These data indicate that superficial layers remap more

232 strongly during novel context exploration and reactivate the original spatial tuning more efficiently

233 during re-exposure to the familiar environment.

234

Next, we assessed whether position along the dorso-ventral axis relates to context

recognition. In contrast to the apparent dorso-ventral gradient of spatial tuning in layer 2/3, we

found no difference in tuning correlation between fam and nov $(P=0.290, N=47$ and 28 dorsal and ventral cells, respectively) or fam and fam' $(P=0.729)$ for dorsally or ventrally located neurons (we restricted this analysis to layer $2 / 3$ recordings from the left hemisphere to exclude inter-hemispheric differences as a confounding factor, SI Appendix, Fig. S6).

Finally, we assessed spatial tuning during nov and fam' in recordings from the left and right mPFC (we restricted the analysis to deep layer neurons to exclude layer differences as a confounding factor). Stability between fam and nov was significantly higher in the left compared to the right $\mathrm{mPFC}(P=0.045, N=142$ and 509 neurons recorded in the left and right mPFC, respectively), suggesting stronger remapping in the right hemisphere. Furthermore, spatial stability

245 between fam and fam' was significantly higher in the right mPFC $\left(P=2.9 * 10^{-8}\right.$, Fig. 5G). Thus, our data suggest that the right mPFC discriminates better between familiar and novel environments and reactivates the original spatial tuning pattern more efficiently upon return to the familiar space.

\section{Discussion}

250 Here we show that the mPFC of mice displays detectable spatial tuning during spontaneous, self251 paced motion, including the representation of position as well as context. In line with findings of 252 grid cells in the human cortex (31) (32) and spatially modulated cells in the cortex of rodents (4) (5) 253 (6) (7) (8), our findings are consistent with a more general neuronal system tracking spatial 254 variables in neocortical columns (33). Our data further demonstrate that in the mPFC distinct 
255 features of spatial representation occur lateralized and along dorso-ventral and superficial-deep 256 radial gradients.

257 The stronger spatial tuning of the dorsal mPFC is surprising since multiple studies 258 demonstrated denser innervation of the ventral PFC by hippocampal inputs (23) (24) (25) (26), 259 which presumably composes the primary source of spatial information to the mPFC (10). Direct 260 projections from the ventral CA1 furthermore terminate in deep cortical layers, particularly in 261 dorsal parts of the mPFC (23) (26). Moreover, optogenetic activation of direct hippocampal 262 afferents to the mPFC revealed stronger synaptic drive to layer 5 than to layer 3 neurons of the 263 prelimbic cortex (26). In addition to direct hippocampal afferents, spatially modulated inputs reach 264 the mPFC via the thalamic nucleus reuniens, which relays hippocampal afferents to the mPFC (14) 265 (34). However, similar to the direct hippocampal inputs, nucleus reuniens afferents have also been 266 shown to preferentially target ventral prefrontal areas (34). Our findings of a dorso-ventral gradient 267 of spatial tuning in superficial but not deep layers of the mPFC is thus not likely explained by direct 268 hippocampal input. In fact, deep layer neurons located ventrally are more strongly entrained by 269 theta oscillations, as one would predict based on the anatomy of hippocampal inputs. In contrast, 270 superficially located neurons are more strongly theta-coupled when they reside in dorsal parts of the 271 mPFC. We thus propose that the spatial tuning of prefrontal neurons is only partly contingent on 272 direct hippocampal input. Lesioning the hippocampus indeed reduced spatial information of 273 neocortical cells and decreased position decoding accuracy based of spatially tuned neocortical 274 neurons but did not entirely eliminate spatial tuning in the neocortex (8), consistent with an 275 additional hippocampus-independent mechanism. Tracing studies confirmed the presence of 276 entorhinal cortex projections in the dorsal and ventral portions of the mPFC (35) (36), which might 277 contribute to spatial tuning properties of mPFC cells. In addition, spatial signals might reach the 278 mPFC via other cortical areas. For instance, prominent spatial tuning features have been observed in 279 the somatosensory cortex (7), which projects monosynaptically to the mPFC (18). Only few 
connections from the hippocampal formation to the somatorsensory cortex have been identified

281 (25), which gave rise to the hypothesis that the somatosensory cortex might contain a hippocampus-

282 independent spatial navigation system (7). It remains to be tested whether the complex topographical nature of prefrontal spatial tuning emerges due to complementary inputs from the hippocampal and somatosensory spatial navigation systems.

Our data revealed a lateralization of spatial representation, with stronger spatial tuning properties in the left but more pronounced familiarity detection in the right mPFC. Prefrontal lateralization has been demonstrated using functional imaging in human participants and showed that hemispheric specialization depends on the nature of the task (37). While left prefrontal regions are considered to be involved in processing the 'verbal domain', the right prefrontal cortex processes visuo-spatial information (27). Furthermore, the left prefrontal cortex has been associated with drawing logical and causal inferences while the right prefrontal area has been implicated in the detection of inconsistencies between pre-existing beliefs and new information (29). In line with the distinct function of the left and right PFC in humans and reports of lateralization in mouse auditory cortex (38) as well as hippocampus (39) (40), we propose a functional division of labor between left and right mPFCs in mice such that the dorsal part of the left prefrontal hemisphere might be best suited to integrate the own position in space, whereas the dorsal right mPFC might support the differentiation between familiarity and novelty (Fig. 5H). The mechanisms of differential spatial tuning properties of both mPFCs remain elusive. Activity in the left but not right CA3 has been reported to be required for spatial memory (40), and long-term plasticity of CA3 outputs in CA1 occur only on synapses originating from the left but not right CA3 (41). While these data suggest pronounced lateralization within the hippocampal circuitry, potential hemispheric asymmetries in

302 CA1 output to the neocortex are much less understood. Based on our data we predict that axons 
304 to the mPFC are ipsilateral), while similarity during context re-exposure will be higher in the right

305 CA1 output.

306 Our data reveal prominent remapping in the mPFC during exploration of a novel arena

307 reminiscent of dynamic changes observed in CA1 pyramidal neurons upon exposure to a novel

308 arena (30) (42). We further find a dissociation of spatial tuning per se and remapping properties

309 such that superficial and deep layers show comparable spatial tuning while superficial layers

310 discriminate contexts more efficiently. Stronger context discrimination in superficial layers might

311 relate to the integration of rich intra-cortical inputs in sparsely active layer $2 / 3$ neurons. Similar to

312 the known high selectivity of superficial layer cells in sensory cortices for stimulus properties (43)

313 (44), context specificity might be the analog of prefrontal responses in layer 2/3 during contextual

314 changes (the environment being the stimulus property in this sense).

315 In summary, our work indicates that in the absence of particular cognitive task demands the

316 prefrontal cortex represents spatial and contextual information in a layer- and hemisphere-

317 dependent manner. Such a division of labor among medial prefrontal cortical subnetworks may be

318 important in supporting local network computation relevant for the execution of context-dependent

319 behavioral outcomes. Further investigations of area-specific mPFC circuits during cognitive tasks,

320 resolving distinct layers, dorso-ventral subregions, as well as the left and right hemisphere's

321 subnetworks will help to understand their contribution to cognitive behavior. 


\section{Materials and Methods}

323 Mice

$324 \mathrm{C} 57 \mathrm{Bl} / \mathrm{J}$ mice of both sexes were maintained on a 12h dark-light cycle with free access to food and

325 water. At the start of the experiment, the animals were 6 to 13 weeks old. All experiments were 326 performed in agreement with national legislation and were approved by the Regierungspräsidium

327 Freiburg.

329 Surgical procedures

330 For single-unit recording in the virtual reality, a stainless steel head plate was implanted on the skull under general anesthesia in isoflurane (induction: 3\%, maintenance: 1-2\%) using dental cement.

332 The animals were allowed to recover from head plate implantation for at least three days. Buprenorphin (0.1 mg/kg body weight) and Carprofen (5 mg/kg body weight) were injected subcutaneously before the surgery for pain relief. Once the animals were habituated to head-fixation (see below), a craniotomy was performed over both mPFCs (1.9 mm anterior, $0.4 \mathrm{~mm}$ lateral of bregma) under isoflurane anesthesia. The craniotomy was then sealed of with QuikCast elastomer until the recordings took place. An additional injection of Carprofen was given for analgesia prior to craniotomy.

$\underline{\text { Single-unit recording in the virtual reality }}$

341 After recovery from head-plate implantation, the mice were trained to run on a circular track in a

342 virtual reality. They were briefly sedated with isoflurane applied in oxygen and head-fixed such that 343 they could comfortably stand on a circular styrofoam weel. First, animals were habituated to head344 fixation without the virtual reality turned on. After at least three days of habituation, training in the 345 virtual reality (circular track, length $1.5 \mathrm{~m}$ or $2 \mathrm{~m}$, visual cues placed outside the arena) was conducted. The virtual reality was constructed with open-source 3D rendering software Blender 
347 (45) and was projected on five computer screens surrounding the head-fixation setup. Over

348 subsequent days, mice were accustomed to the virtual environment until the animals appeared calm

349 and traversed the circular maze reliably.

Recordings were performed in the arena to which the animals were habituated ( $N=17$ mice mPFC, $N=2$ mice CA1). The animals completed on average $21.8 \pm 5.3$ laps per recording session. In a subset of mice ( $N=8$ animals), we additionally exposed the animals within the same session to a novel arena (nov, $13.8 \pm 2.2$ laps completed), which the mice encountered for the first time during

354 the initial recording session. The novel arena differed in size (3 m circle length) and visual features

355 from the familiar arena. After recording in the novel arena, the animals were again exposed to the familiar track to assess the reactivation of spatially tuned activities in the familiar context $(15.4 \pm$ 4.3 laps were completed on average).

Recordings were conducted 1-3 days after the craniotomy. A single-shank silicon probe with 64 recording sites (electrode pitch $20 \mu \mathrm{m}$, total shank length $1275 \mu \mathrm{m}$, H3 probe, Cambridge Neurotech) coated with a fluorescent marker ( $\mathrm{DiI}$ or $\mathrm{DiO}$ ) was slowly ( 2-5 $\mu \mathrm{m} / \mathrm{s}$ ) lowered to the mPFC (1800-1900 $\mu \mathrm{m}$ below brain surface). In three mice, a four shank probe with 16 electrodes per shank (Cambridge Neurotech) was used to perform simultaneous recordings from different neocortical layers. After insertion, the probe was left in place before starting the recording for 10-15 min. In two mice, recordings were obtained from hippocampal CA1. Wide-band neural signals were recorded at $30 \mathrm{kHz}$ sampling with a 64-channel amplifier (Intan Technologies) connected to an USB acquisition board (OpenEphys). The position in the virtual reality was recorded as a pulse-width modulated signal using one of the analog input channels of the OpenEphys USB interface board. After recording, the silicon probe was slowly retracted and the craniotomy sealed off with

369 QuickCast elastomer. 1-2 recording sessions were performed with each animal, with one session per 370 day. 


\section{Histology}

373 After the last recording session, the animals were deeply anesthetized with an intraperitoneal

374 injection of ketamin/xylazine and transcardially perfused with $\sim 20 \mathrm{ml}$ phosphate-buffered saline

375 followed by $\sim 30 \mathrm{ml}$ of $4 \%$ paraformaldehyde. After post-fixation over night, $100 \mu \mathrm{m}$-thick frontal

376 sections of the mPFC were cut, washed several times in phosphate-buffered saline (PBS) and

377 stained with 4',6-diamidino-2-phenylindole. The location of the silicon probe was visualized with a

378 laser-scanning microscope (LSM 710 or 900, Zeiss) following the nomenclature of the Allen Brain

379 Atlas. To determine laminar depth of the probe, the distance from pia to the shank was measured

380 orthogonal to the cortical layers.

382 Single-unit isolation

383 Single unit clusters were isolated from bandpass-filtered raw data $(0.3-3 \mathrm{kHz})$ using

384 MountainSort (46). Putative single-unit clusters that fulfilled quality criteria of high isolation index

385 (>0.90) and low noise overlap $(<0.1)$ were kept for manual curation, during which only clusters

386 with a clear refractory period and clean waveform shape were saved for further analysis. In case of

387 two clusters with similar waveforms, cross-correlation was used to assess whether clusters had to be

388 merged. Isolated units were separated in putative excitatory and inhibitory neurons based on trough-

389 to-peak duration and asymmetry index (47) using k-means clustering. To quantify the stability of

390 single-unit recording during the recording sessions, waveforms of the channel with the largest

391 voltage deflection were average separately for the time the animal explored the familiar track and

392 during re-exposure to the familiar track after completion of running on the novel track. Stability was

393 assessed by calculating Pearson's correlation coefficient between the waveforms of each unit during

394 both conditions and by correlating the peak amplitude recorded during both sessions. To extract the

395 physical location of the units, the channel on the silicon probe with the largest negative amplitude 
396

397 398

399

400

401

402

403

404

405

406

407

where $N$ is the number of bins, $p$ is the occupation probability in the $\mathrm{i}^{\text {th }}$ bin, $f_{i}$ is the activity in bin $i$, and $\mathrm{f}$ is the average activity of the neuron across bins (8). A control data set was constructed for each recording session by shuffling the inter-spike intervals of each neuron before spatial binning. To determine spatial consistency within the session, average occupancy-normalized spatial histograms of spikes were constructed separately for the first and last six runs of a given recording session. Consistency was then defined by Pearson's correlation coefficient between both histograms and computed for each unit. For spatial stability, average spike rates as a function of position on the maze (40 bins) were obtained for fam, nov and fam'. Stability was defined as Pearson's $r$ computed 420 for the average spatial maps. Control data were generated by shuffling the interspike intervals of 
421 each neuron. In case of single-run correlations, Pearson's r was computed separately for all possible

422 run pairs within fam, between fam and nov, or between fam and fam' in a round-robin fashion and

423 averaged across runs.

424 Theta coupling was assessed by first extracting the phase of the 6-12 Hz-filtered local field 425 potential of all spikes during running, selecting cells with non-uniform phase distributions using 426 Rayleigh’s test, and computing the pairwise phase consistency $P P C$ as

$$
P P C=\frac{\pi-2 D}{\pi},
$$

where $D$ is the average pairwise circular distance between individual spikes as

$$
D=\frac{2}{N(N-1)} \sum_{j=1}^{N-1} \sum_{k=(j+1)}^{N} d\left(\theta_{j}, \theta_{k}\right) \text {, }
$$

where $\theta_{j}$ and $\theta_{k}$ are the phases of the local field potential of spikes $j$ and $k$, and $N$ is the total number of spikes of the unit. Theta power was computed using Welch's method with a window size of $10 \mathrm{~s}$ 432 and 10x zero-padding.

\section{$\underline{\text { Principal component analysis }}$}

Binned spike trains of single units (bin width $30 \mathrm{~ms}$ ) were square-root transformed and smoothed with a Gaussian kernel of s.d. $50 \mathrm{~ms}$ (48). Neurons with low mean firing rate $<1 \mathrm{~Hz}$ were excluded from this analysis (48). A control data set was constructed for each recording session by shuffling the inter-spike intervals of each pyramidal neuron before binning. For each session, principal component analysis was then applied to the $N$ by $B$ array containing spike data of $N$ neurons in $B$ bins, thus resulting in $N$ principal components. To obtain time-resolved population dynamics, the smoothed spike trains were projected onto the principal components $C$, giving a $C \mathrm{x}$

$442 B$ array of population dynamics over time. Each component vector was binned as a function of 443 location for each run on the maze (40 bins). To determine spatial variance, all runs of a session were 
445 population activity was determined and averaged across components, weighted by the explained

446 variance of the respective component. To obtain spatial consistency, the first and last six runs were

447 averaged separately for the leading ten components. Then, for each component, the spatial

448 correlation was determined as Pearson's correlation coefficient. Lastly, the spatial correlation was

449 averaged across components, while weighting by the explained variance of each component. To

450 determine single run consistency, we computed the spatial correlation between individual runs that

451 occurred between one and ten runs apart from each other. Correlations between all possible

452 combinations of run pairs in each distance category were averaged separately for each component.

453 Finally, an average across the leading ten components was obtained while weighting with the

454 explained variance of the component. The same analysis was also performed for individual units by

455 taking their binned firing as a basis for single-trial correlation.

\section{$\underline{\text { Statistical analysis }}$}

458 Sample sizes for most comparisons of single unit data were large $(>N=50)$. Comparisons were

459 done with two-sided Welch's tests, which is robust against deviation from normal distribution at 460 large sample sizes (49). In cases of smaller sample sizes, comparisons were performed with a 461 Mann-Whitney U-test if the data did not follow a normal distribution, otherwise an unpaired $t$-test 462 was used. Normality was assessed with a Shapiro-Wilk test. Correlations between spatial tuning 463 functions were tested with Pearson's correlation coefficient to detect linear correlations. 464 Correlations for which linearity could not be assumed (e.g. cortical depth vs. spatial information) 465 were assessed with Spearman's correlation coefficient. Pairwise comparisons of spatial tuning 466 parameters for simultaneous recordings were done with a paired $t$-test. Significant theta coupling 467 was assessed with Rayleigh’s test for circular uniformity. Data are presented as full data ranges with 468 means (violin plots) or as mean \pm sem where indicated. All analysis (except for initial spike sorting, 469 see above) including statistics were performed in Python2.7. 
bioRxiv preprint doi: https://doi.org/10.1101/2021.06.04.447085; this version posted June 4,2021 . The copyright holder for this preprint (which was not certified by peer review) is the author/funder, who has granted bioRxiv a license to display the preprint in perpetuity. It is made available under aCC-BY-NC-ND 4.0 International license.

\section{Acknowledgments}

471 We thank Johannes Letzkus, Jozsef Csicsvari and Antje Kilias for critical comments on earlier

472 versions of the manuscript, and Kerstin Semmler and Karin Winterhalter for technical assistance.

\section{References}

1. C. Wang, X. Chen, J. J. Knierim, Egocentric and allocentric representations of space in the rodent brain. Curr. Opin. Neurobiol. 60, 12-20 (2020).

2. T. Hafting, M. Fyhn, S. Molden, M.-B. Moser, E. I. Moser, Microstructure of a spatial map in the entorhinal cortex. Nature 436, 801-806 (2005).

3. T. Solstad, C. N. Boccara, E. Kropff, M.-B. Moser, E. I. Moser, Representation of Geometric Borders in the Entorhinal Cortex. Science 322, 1865-1868 (2008).

4. C. D. Harvey, P. Coen, D. W. Tank, Choice-specific sequences in parietal cortex during a virtual-navigation decision task. Nature 484, 62-68 (2012).

5. D. Mao, S. Kandler, B. L. McNaughton, V. Bonin, Sparse orthogonal population representation of spatial context in the retrosplenial cortex. Nat Commun 8, 243 (2017).

6. A. Fiser, et al., Experience-dependent spatial expectations in mouse visual cortex. Nat Neurosci 19, 1658-1664 (2016).

7. X. Long, S.-J. Zhang, A novel somatosensory spatial navigation system outside the hippocampal formation. Cell Research, 1-15 (2021).

8. I. M. Esteves, et al., Spatial Information Encoding across Multiple Neocortical Regions Depends on an Intact Hippocampus. J Neurosci 41, 307-319 (2021).

9. A. Diamond, Executive Functions. Annual Review of Psychology 64, 135-168 (2013).

10. T. Spellman, et al., Hippocampal-prefrontal input supports spatial encoding in working memory. Nature 522, 309-314 (2015).

11. J. J. Tuscher, L. R. Taxier, A. M. Fortress, K. M. Frick, Chemogenetic inactivation of the dorsal hippocampus and medial prefrontal cortex, individually and concurrently, impairs object recognition and spatial memory consolidation in female mice. Neurobiol Learn Mem 156, 103-116 (2018).

12. M. C. Zielinski, J. D. Shin, S. P. Jadhav, Coherent Coding of Spatial Position Mediated by Theta Oscillations in the Hippocampus and Prefrontal Cortex. J. Neurosci. 39, 4550-4565 (2019).

13. S. Fujisawa, A. Amarasingham, M. T. Harrison, G. Buzsáki, Behavior-dependent short-term assembly dynamics in the medial prefrontal cortex. Nat. Neurosci. 11, 823-833 (2008). 
14. H. T. Ito, S.-J. Zhang, M. P. Witter, E. I. Moser, M.-B. Moser, A prefrontal-thalamohippocampal circuit for goal-directed spatial navigation. Nature 522, 50-55 (2015).

15. M. W. Jung, Y. Qin, B. L. McNaughton, C. A. Barnes, Firing characteristics of deep layer neurons in prefrontal cortex in rats performing spatial working memory tasks. Cerebral Cortex (New York, N.Y.: 1991) 8, 437-450 (1998).

16. K. Kaefer, M. Nardin, K. Blahna, J. Csicsvari, Replay of Behavioral Sequences in the Medial Prefrontal Cortex during Rule Switching. Neuron (2020) https:/doi.org/10.1016/j.neuron.2020.01.015.

17. R. J. Douglas, K. A. C. Martin, Neuronal circuits of the neocortex. Annu Rev Neurosci 27, 419-451 (2004).

18. S. Ährlund-Richter, et al., A whole-brain atlas of monosynaptic input targeting four different cell types in the medial prefrontal cortex of the mouse. Nat Neurosci 22, 657-668 (2019).

19. K. L. Agster, R. D. Burwell, HIPPOCAMPAL AND SUBICULAR EFFERENTS AND AFFERENTS OF THE PERIRHINAL, POSTRHINAL, AND ENTORHINAL CORTICES OF THE RAT. Behav Brain Res 254, 50-64 (2013).

20. A. J. Hill, First occurrence of hippocampal spatial firing in a new environment. Exp Neurol 62, 282-297 (1978).

21. M. A. Wilson, B. L. McNaughton, Dynamics of the hippocampal ensemble code for space. Science 261, 1055-1058 (1993).

22. C. Gemmell, M. Anderson, S. M. O’Mara, Deep layer prefrontal cortex unit discharge in a cue-controlled open-field environment in the freely-moving rat. Behavioural Brain Research 133, 1-10 (2002).

23. T. M. Jay, M. P. Witter, Distribution of hippocampal CA1 and subicular efferents in the prefrontal cortex of the rat studied by means of anterograde transport of Phaseolus vulgarisleucoagglutinin. J. Comp. Neurol. 313, 574-586 (1991).

24. F. Condé, E. Maire-Lepoivre, E. Audinat, F. Crépel, Afferent connections of the medial frontal cortex of the rat. II. Cortical and subcortical afferents. J. Comp. Neurol. 352, 567-593 (1995).

25. L. A. Cenquizca, L. W. Swanson, Spatial organization of direct hippocampal field CA1 axonal projections to the rest of the cerebral cortex. Brain Res Rev 56, 1-26 (2007).

26. X. Liu, A. G. Carter, Ventral Hippocampal Inputs Preferentially Drive Corticocortical Neurons in the Infralimbic Prefrontal Cortex. J. Neurosci. 38, 7351-7363 (2018).

27. A. D. Wagner, et al., Material-specific lateralization of prefrontal activation during episodic encoding and retrieval. Neuroreport 9, 3711-3717 (1998).

28. W. M. Kelley, et al., Hemispheric specialization in human dorsal frontal cortex and medial temporal lobe for verbal and nonverbal memory encoding. Neuron 20, 927-936 (1998). 
29. V. Goel, "Chapter 10 - Hemispheric asymmetry in the prefrontal cortex for complex cognition" in Handbook of Clinical Neurology, The Frontal Lobes., M. D’Esposito, J. H. Grafman, Eds. (Elsevier, 2019), pp. 179-196.

30. T. Hainmueller, M. Bartos, Parallel emergence of stable and dynamic memory engrams in the hippocampus. Nature 558, 292-296 (2018).

31. C. F. Doeller, C. Barry, N. Burgess, Evidence for grid cells in a human memory network. Nature 463, 657-661 (2010).

32. J. Jacobs, et al., Direct recordings of grid-like neuronal activity in human spatial navigation. Nature Neuroscience 16, 1188-1190 (2013).

33. J. Hawkins, M. Lewis, M. Klukas, S. Purdy, S. Ahmad, A Framework for Intelligence and Cortical Function Based on Grid Cells in the Neocortex. Front. Neural Circuits 12 (2019).

34. G. V. Di Prisco, R. P. Vertes, Excitatory actions of the ventral midline thalamus (rhomboid/reuniens) on the medial prefrontal cortex in the rat. Synapse 60, 45-55 (2006).

35. W. B. Hoover, R. P. Vertes, Anatomical analysis of afferent projections to the medial prefrontal cortex in the rat. Brain Struct Funct 212, 149-179 (2007).

36. B. Delatour, M. P. Witter, Projections from the parahippocampal region to the prefrontal cortex in the rat: evidence of multiple pathways. The European Journal of Neuroscience 15, 14001407 (2002).

37. K. E. Stephan, et al., Lateralized Cognitive Processes and Lateralized Task Control in the Human Brain. Science 301, 384-386 (2003).

38. R. B. Levy, et al., Circuit asymmetries underlie functional lateralization in the mouse auditory cortex. Nature Communications 10, 2783 (2019).

39. Y. Shinohara, et al., Left-right asymmetry of the hippocampal synapses with differential subunit allocation of glutamate receptors. PNAS 105, 19498-19503 (2008).

40. D. Song, et al., The lateralization of left hippocampal CA3 during the retrieval of spatial working memory. Nature Communications 11, 2901 (2020).

41. O. A. Shipton, et al., Left-right dissociation of hippocampal memory processes in mice. PNAS 111, 15238-15243 (2014).

42. A. Rubin, N. Geva, L. Sheintuch, Y. Ziv, Hippocampal ensemble dynamics timestamp events in long-term memory. eLife 4, e12247 (2015).

43. K. Ohki, S. Chung, Y. H. Ch’ng, P. Kara, R. C. Reid, Functional imaging with cellular resolution reveals precise micro-architecture in visual cortex. Nature 433, 597-603 (2005).

44. M. L. Andermann, C. I. Moore, A somatotopic map of vibrissa motion direction within a barrel column. Nat Neurosci 9, 543-551 (2006).

45. C. Schmidt-Hieber, M. Häusser, Cellular mechanisms of spatial navigation in the medial entorhinal cortex. Nat. Neurosci. 16, 325-331 (2013). 
46. J. E. Chung, et al., A Fully Automated Approach to Spike Sorting. Neuron 95, 1381-1394.e6 (2017).

47. A. Sirota, et al., Entrainment of neocortical neurons and gamma oscillations by the hippocampal theta rhythm. Neuron 60, 683-697 (2008).

48. J. A. Gallego, M. G. Perich, R. H. Chowdhury, S. A. Solla, L. E. Miller, Long-term stability of cortical population dynamics underlying consistent behavior. Nat. Neurosci. 23, 260-270 (2020).

49. J. M. Stonehouse, G. J. Forrester, Robustness of the $t$ and U tests under combined assumption violations. Journal of Applied Statistics 25, 63-74 (1998). 


\section{Figures and figure legends}
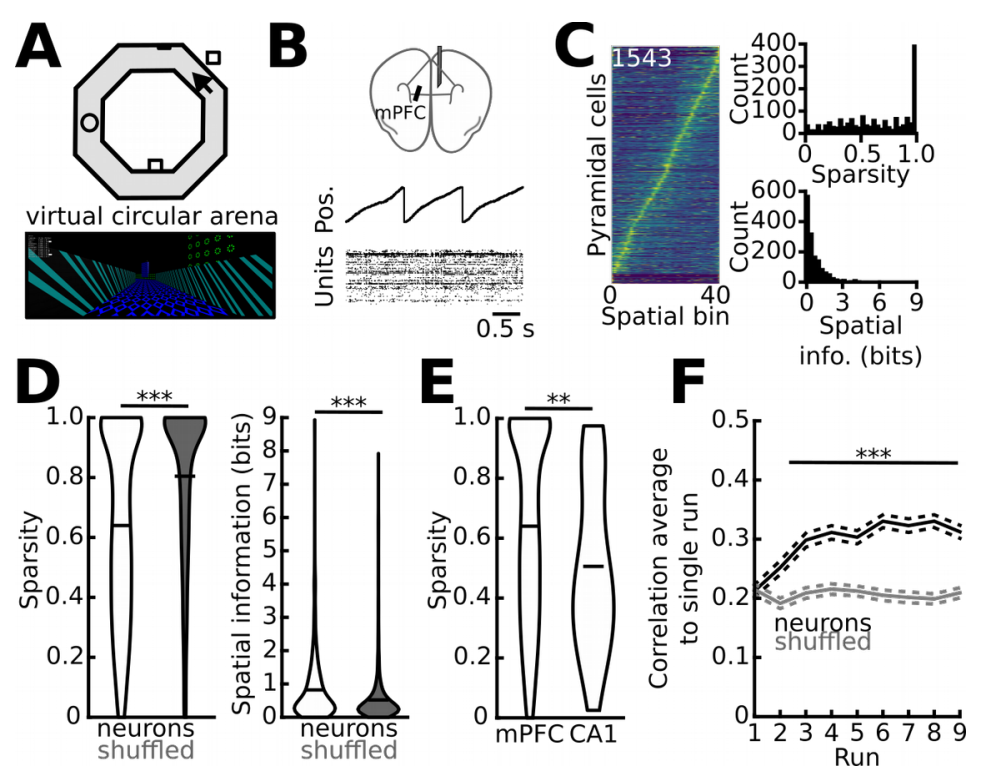

Figure 1: Spatially tuned activity of prefrontal pyramidal neurons during self-paced, unrewarded motion.

(A) Experimental protocol for head-fixed recordings during navigation in the virtual reality. Arrow on the track

indicates the position shown at the bottom. (B) Single-unit recording during movement on the track. Black line indicates position on the track. Each row of the raster plot denotes one unit. (C) Left: Spatially binned activity of pyramidal cells ( $N=17$ mice). Right: Histograms of sparsity and spatial information. Low sparsity values indicate stronger spatial tuning. (D) Pyramidal cells show significant spatial tuning compared to shuffled controls. Sparsity: $P=4 * 10^{-55}$. Spatial information: $P=10^{-21}$. $N=1543$ units, Welch's tests. (E) Comparison of sparsity between mPFC and CA1 pyramidal cells ( $N=35$ units). $P=0.002$, Mann-Whitney U-test. (F) Correlation of average spatial tuning to individual runs reveals significant spatial representation from the second lap onwards (run 1: $P=0.850$, run 2-10: $P=10^{-5}$ to $P=10^{-20}$, Welch's tests). ${ }^{* *} P<0.01,{ }^{* * *} P<0.001$. Lines in (D) and (E) show the mean. Data in (F) are mean and sem. 

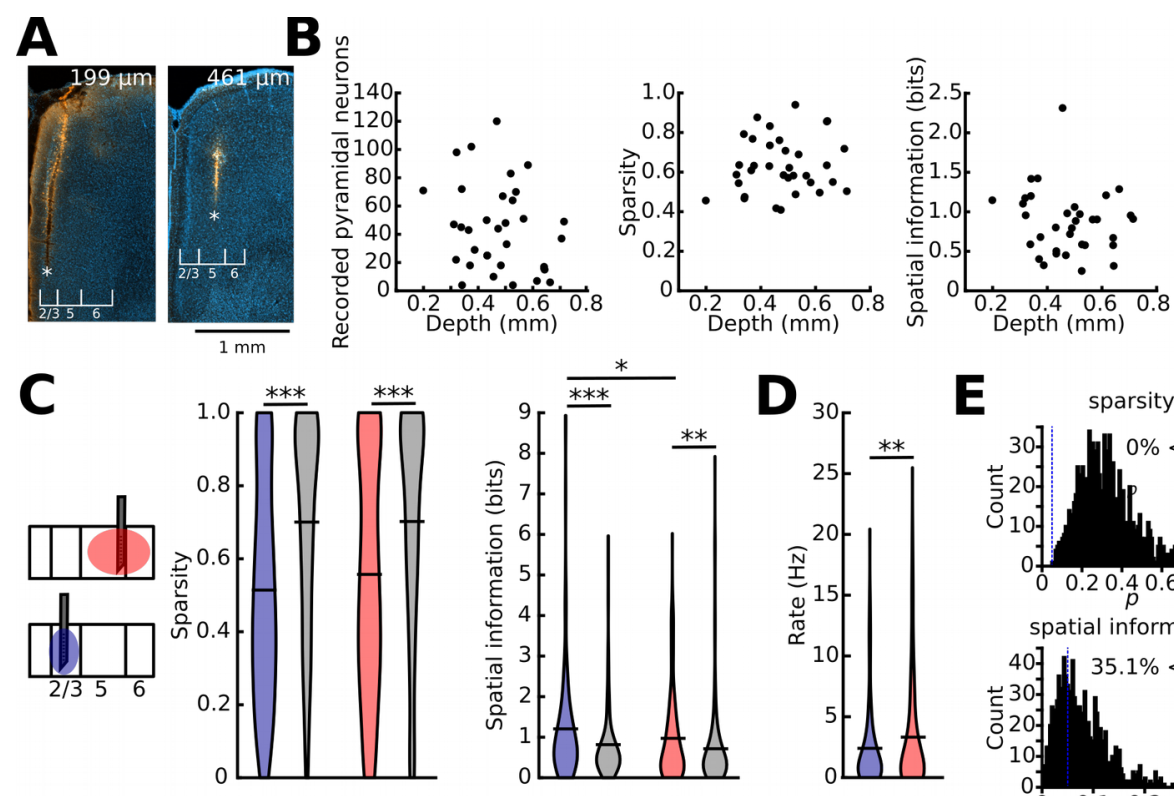

E
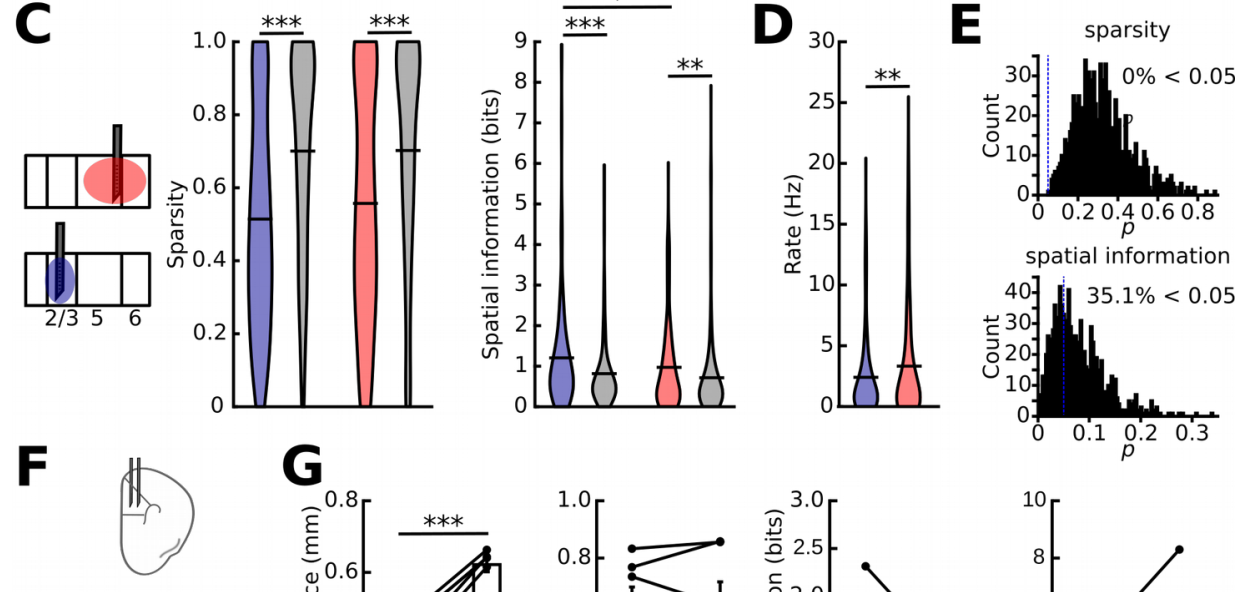

\section{G}
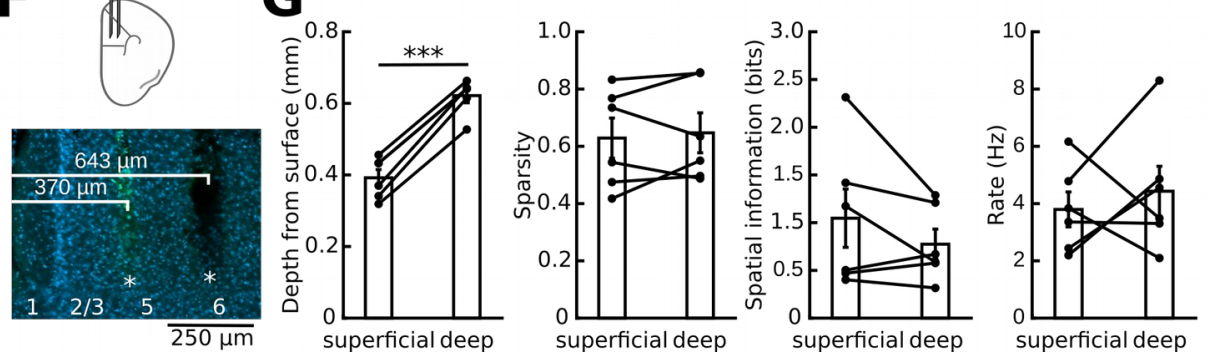

Figure 2: Spatial tuning emerges across cortical layers. (A) Histology of probe tracks located in superficial layer 2/3 (left) and deep layer 5 (right). Values in the top right corner indicate the laminar depth of the recording. (B) The number of recorded pyramidal neurons (left, $P=0.198$ ), average sparsity (middle, $P=0.621$ ), and average spatial information (right, $P=0.257$ ) did not correlate with recording depth. (C) Pyramidal neurons in layer 2/3 and layer 5/6 show significant sparsity and spatial information compared to shuffled controls (layer $2 / 3$ : sparsity: $P=1.6 * 10^{-11}$, spatial information: $P=0.0004, N=233$ neurons. Layer 5/6: sparsity: $P=1.3^{*} 10^{-8}$, spatial information: $P=0.002, N=311$ neurons, shuffled data in grey). Comparisons between layer $2 / 3$ and 5/6: sparsity: $P=0.112$, spatial information: $P=0.03$. (D) Firing rate was significantly higher in deep layers. $P=0.007$. (E) Rate-matched iterations of sparsity (top) and spatial information (bottom) revealed no significant differences in the majority of cases. (F) Top, schematic of simultaneous recordings with two shanks placed at different cortical depths. Bottom, example of dual-site recording at 370 and 643 $\mu \mathrm{m}$ depth. (G) Left, summary of recording depth of the shanks. Right, there was no differences in sparsity ( $P=0.629$ ), spatial information $(P=0.204)$ or firing rate $(P=0.562)$ of neurons recorded on superficial and deep shanks. Values are session averages for both shanks. Depth comparison: $P=1.4 * 10^{-5}$. $N=6$ sessions from 3 mice. $* P<0.05, * * P<0.01$, 
A
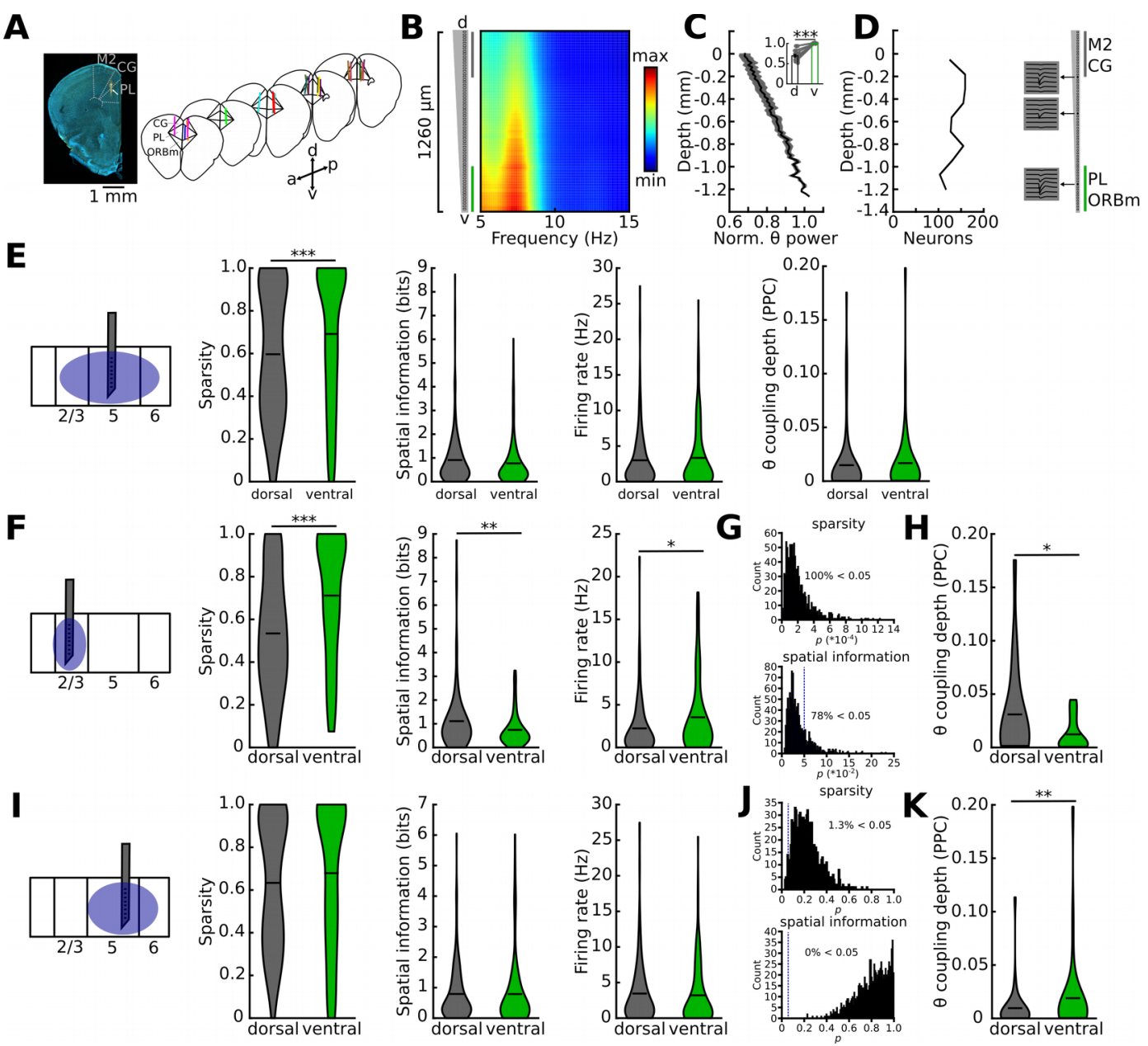

Figure 3: A layer-specific dorso-ventral gradient in spatial representation. (A) Reconstruction of recording sites.

Left: DiI-labelled probe track in the prefrontal cortex (arrow). Right: Location of silicon probes. Each mouse is presented in a different color ( $N=14$ mice). M2: accessory motor cortex, PL: prelimbic cortex, CG: cingulate cortex, ORBm: medial orbital cortex, a: anterior, p: posterior, d: dorsal, v: ventral. (B) Example of the dorsoventral depth profile of theta power along the shank. (C) Average normalized theta power as a function of d-v depth. Inset: Average normalized theta power of the dorsal five versus the ventral five recording sites. $P=1.2 * 10^{-9}$, paired $t$-test. (D) Number of recorded neurons along the shank length (10 bins). Right: Identification of unit location along the probe shank. Grey and green depths indicate the locations used for grouping neurons into dorsal and ventral mPFC, respectively. (E) Comparisons of sparsity $(P=0.0002)$, spatial information $(P=0.094)$, firing rate $(P=0.326)$ and theta coupling depth ( $P=0.7)$ for the top and bottom $300 \mu \mathrm{m}$ of the shank. PPC: pairwise phase consistency. $N=337$ dorsal and 265 ventral neurons. Welch's tests. (F) The same comparisons for layer 2/3 recordings revealed a prominent d-v gradient in sparsity $\left(P=6.4 * 10^{-6}\right)$ and spatial information $(P=0.007)$ and a difference in firing rate $(P=0.011) . \mathrm{N}=125$ dorsal and 104 ventral neurons. Welch’s tests. (G) Rate-matched iteration test revealed significant $\mathrm{d}-\mathrm{v}$ gradients for sparsity and spatial

514 information in the majority of iterations. (H) A d-v gradient in theta coupling strength. $P=0.035, N=18$ dorsal and 31 

made available under aCC-BY-NC-ND 4.0 International license.

515 ventral neurons. Mann-Whitney U-test. (I-K) Same as (F-G), but for deep layer recordings. There was no significant d-v

516 gradient in sparsity $(P=0.176)$, spatial information $(P=0.972)$ or rate $(P=0.592) . N=212$ dorsal and 161 ventral neurons.

517 Welch's tests. Theta coupling depth was significantly higher in ventral vs. dorsal neurons ( $P=0.003)$. Mann-Whitney U-

518 test. $\mathrm{N}=56$ dorsal and 60 ventral neurons. ${ }^{*} P<0.05,{ }^{* *} P<0.01$, ${ }^{* * *} P<0.001$. Lines in (E), (F), (H), (I), and (K) show the

519 mean. Data in (C) are mean and sem. 
A

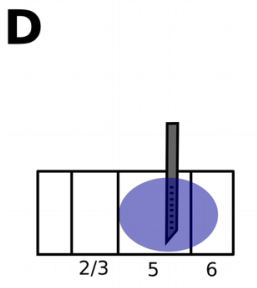

$\mathbf{F}$

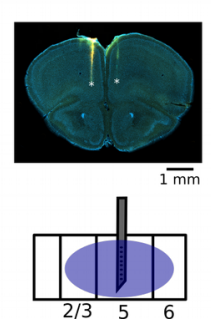

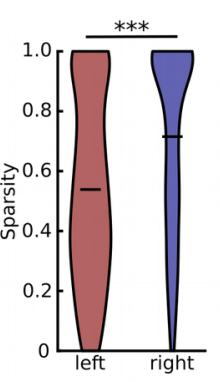
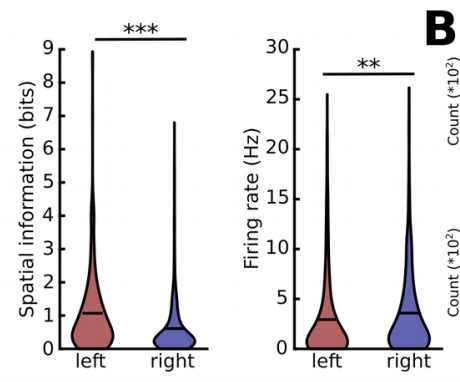

B
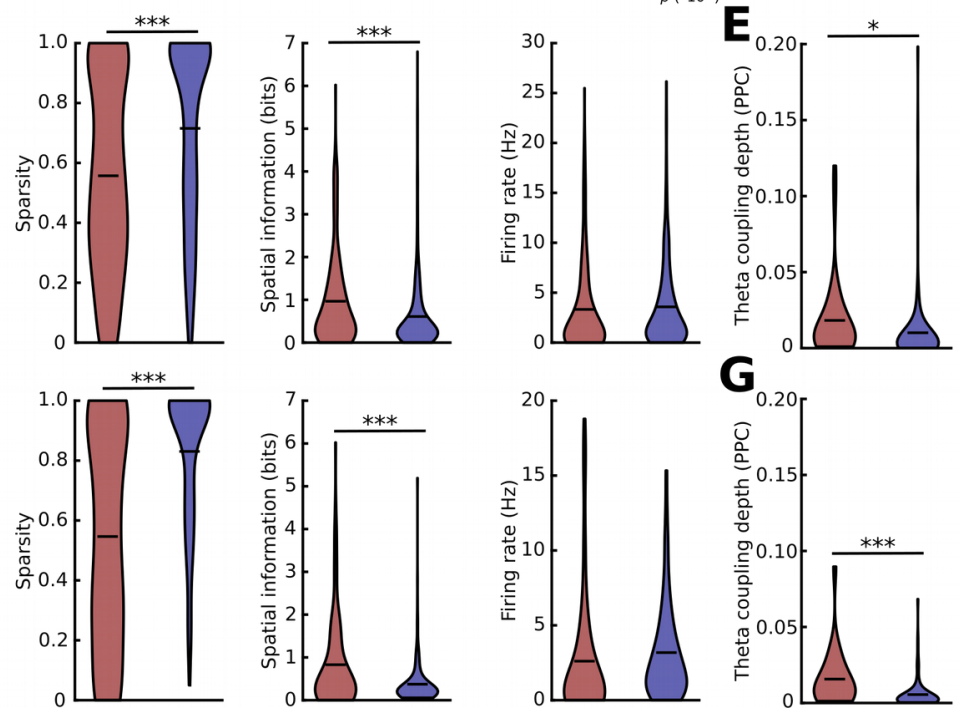

Figure 4: Stronger spatial tuning in the left hemisphere. (A) Pyramidal neurons recorded in the left mPFC showed

lower sparsity $\left(P=8 * 10^{-22}\right)$, higher spatial information $\left(P=4 * 10^{-13}\right)$, and lower firing rates $(P=0.008)$. $\mathrm{N}=544$ pyramidal cells from the left and N=639 from the right, 11 and 8 mice, Welch's tests. (B) Rate-matched iterations to account for firing rate differences revealed significantly different sparsity and spatial information in all cases (1000 iterations). (C) Pyramidal cells of the left hemisphere showed stronger coupling to theta oscillations ( $P=0.004$, Welch's test). $N=100$ left and 203 right neurons. (D) Left-dominant spatial representation was evident in deep layer recordings. Sparsity: $P=2 * 10^{-12}$, Spatial information: $P=4 * 10^{-7}$, Firing rate: $P=0.407$, Welch's tests. $N=311$ pyramidal cells from the left and $N=639$ from the right, 7 and 8 mice. (E) Same as (C) but for layer 5/6 recordings. $P=0.046$, Welch's test. N=61 left and 203 right. (F) Left: Probe tracks of recordings from the left and right mPFC during subsequent sessions from the same animal. Right: Left-dominant spatial representation was evident when restricting the analysis to animals in which sequential left-right or right-left recordings were performed. Sparsity: $P=1 * 10^{-14}$, Spatial information: $P=5^{*} 10^{-6}$, Firing rate: $P=0.167$, Welch's tests. $N=142$ pyramidal cells from the left and $N=213$ from the right, 3 animals. (G) Same as (E) but for sequential recordings. $P=3^{*} 10^{-5} . N=23$ left and 96 right neurons. Mann-Whitney U-test. ${ }^{*} P<0.05, * * P<0.01$, $* * * P<0.001$. Lines in violin plots show the mean. 

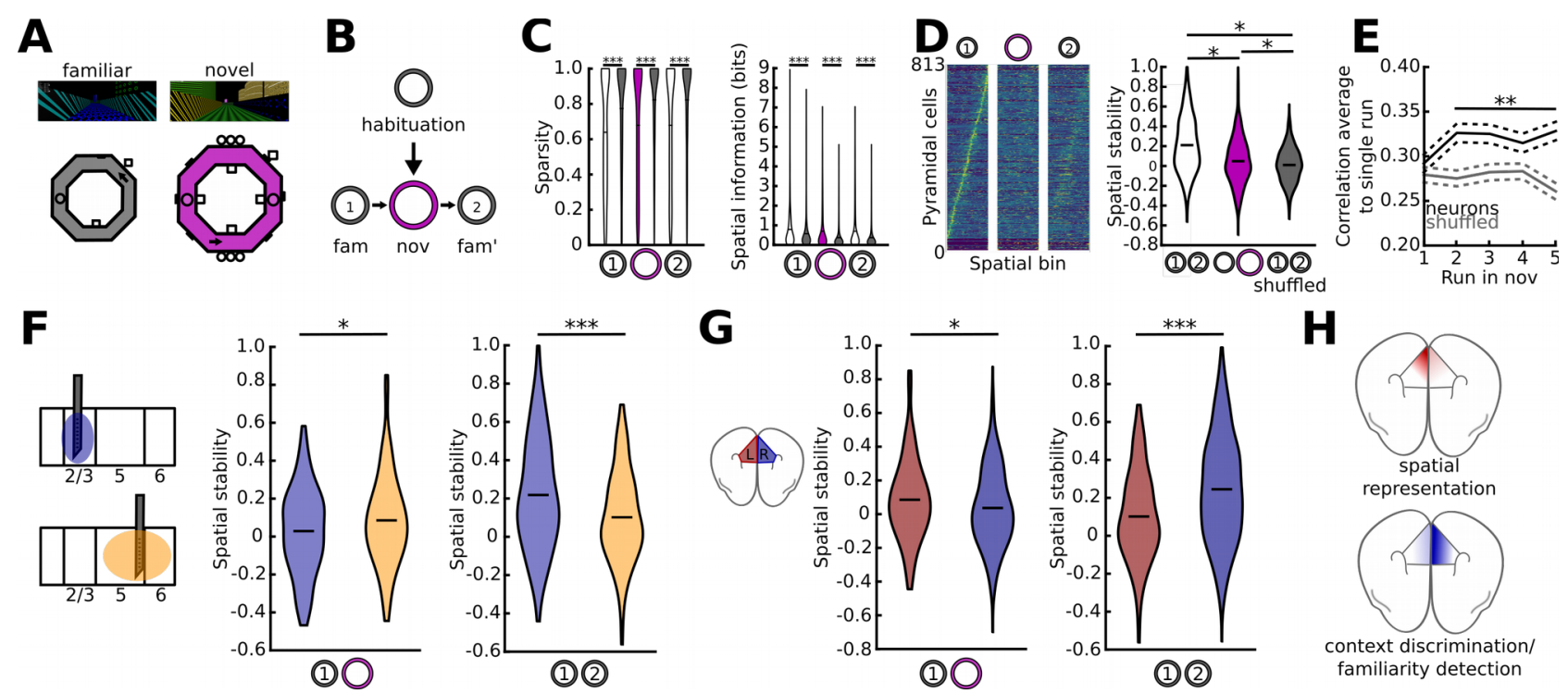

$\mathbf{H}$

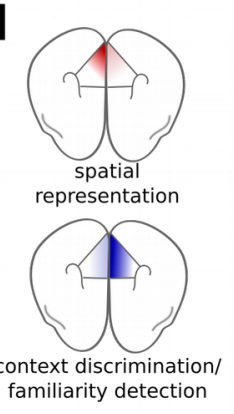

Figure 5: Multiple topographical gradients of spatial pattern reactivation during re-exposure to a familiar environment.

(A) Animals were sequentially exposed to a familiar (left, grey) and novel track (right, purple). Bottom: Schematic of the two tracks. Arrows indicate position of the views shown on top. (B) Schematic of the recording paradigm. (C) Sparsity (values for fam, nov and fam': $\left.P=10^{-22}, 10^{-20}, 10^{-20}\right)$ and spatial information $\left(P=10^{-5}, 10^{-13}, 10^{-10}\right)$ compared to shuffled controls (grey, $N=813$ units from 8 mice, Welch's tests). (D) Left: Spatial maps of all pyramidal cells during fam, nov and fam' sorted by position of peak activity during fam. Plots are shown with the same sorting. Right: Spatial stability (i.e. spatial correlation) between fam and fam', fam and nov, and for shuffled spike trains ( $N=813$ units form 8 mice, Tukey's tests). (E) Correlation of average spatial tuning to individual runs during nov revealed significant spatial representation from the second lap onwards (run 1: $P=0.336$, run 2-5: $P=0.002$ to $P=1.2^{*} 10^{-6}$, Welch's tests). (F)

Spatial stability is higher between fam and nov $(P=0.047)$ but lower between fam and fam' $(\mathrm{P}=0.0003)$ in layer $5 / 6$ compared to layer 2/3. $N=162$ superficial and 142 deep neurons, Welch's tests. (G) Higher spatial stability to nov in the left $(P=0.045)$ but stronger reactivation of spatial tuning during reexposure to fam' in the right hemisphere $\left(P=2.9 * 10^{-8}\right)$. $N=142$ left and 509 right neurons, Welchs tests. (H) Schematic of the proposed role of prefrontal subfields: Dorsal areas of the left mPFC contribute more strongly to spatial representation, while superficial layers of the right mPFC support context discrimination and familiarity detection. ${ }^{*} P<0.05$, ${ }^{* *} P<0.01$, ${ }^{* * *} P<0.001$. Lines in violin plots show the mean. 


\section{Author contributions}

552 J.-F. S. and M.B. designed the study, J.-F. S. performed experiments and analyzed the data, J.-F. S.

553 and M.B. wrote the manuscript.

554

\section{Declaration of interests}

556 The Authors declare no competing interests. 